\title{
In situ observation of cracking and self-healing of solid electrolyte interphases during lithium deposition
}

\section{Tingting Yang}

Yanshan University

Hui Li

Yanshan University

\section{Yongfu Tang}

Yanshan University https://orcid.org/0000-0002-6318-3110

Jingzhao Chen

Nano Energy Center, State Key Laboratory of Metastable Materials Science and Technology, Yanshan University, Qinhuangdao 066004

Hongjun Ye

Nano Energy Center, State Key Laboratory of Metastable Materials Science and Technology, Yanshan University, Qinhuangdao 066004

\section{Baolin Wang}

Georgia Institute of Technology

\section{Yin Zhang}

Georgia Institute of Technology

\section{Congcong Du}

Yanshan University

Jingming Yao

Yanshan University

\section{Baiyu Guo}

Yanshan University

\section{Tong Shen}

Yanshan University https://orcid.org/0000-0002-0391-3361

\section{Liqiang Zhang}

Yanshan University

\section{Ting Zhu}

Georgia Institute of Technology https://orcid.org/0000-0001-8612-9689

Jianyu Huang ( $\sigma$ jhuang@ysu.edu.cn )

Yanshan University https://orcid.org/0000-0002-8424-5368 
Article

Keywords: lithium (Li) whiskers, Li batteries, lithium deposition

Posted Date: December 28th, 2020

DOI: https://doi.org/10.21203/rs.3.rs-124626/v1

License: (c) (i) This work is licensed under a Creative Commons Attribution 4.0 International License. Read Full License 


\section{Abstract}

The growth of lithium $(\mathrm{Li})$ whiskers is detrimental to Li batteries. However, it remains a challenge to directly track Li whisker growth. Here we report in situ observations of electrochemically induced Li deposition under a $\mathrm{CO} 2$ atmosphere inside an environmental transmission electron microscope. We find that the morphology of individual Li deposits is strongly influenced by the competing processes of cracking and self-healing of the solid electrolyte interphase (SEI). When cracking overwhelms self-healing, the directional growth of Li whiskers predominates. In contrast, when self-healing dominates over cracking, the isotropic growth of round Li particles prevails. The Li deposition rate and SEl constituent can be tuned to control the Li morphologies. We reveal a new "weak-spot" mode of Li dendrite growth, which is attributed to the operation of the Bardeen-Herring growth mechanism in the whisker's cross section. This work has implications for the control of Li dendrite growth in Li batteries.

\section{Introduction}

Practical applications of metallic lithium ( $\mathrm{Li}$ ) anodes are considered as the Holy Grail for Li-based batteries due to their ultrahigh theoretical capacity $\left(3860 \mathrm{mAh} \mathrm{g}^{-1}\right) \cdot{ }^{1-12}$ However, the growth of dendritic or mossy Li causes capacity fading, electrolyte consumption, internal short-circuiting, and even fire or explosion of rechargeable Li metal batteries (LMBs). ${ }^{13-22}$ Enormous effort has been devoted to suppressing the dendritic Li growth by introducing additives in electrolytes, ${ }^{23,}{ }^{24}$ increasing electrolyte concentrations, ${ }^{25,26}$ using solid-state electrolytes, ${ }^{27,28}$ constructing artificial solid electrolyte interphases (SEls), ${ }^{29,} 30$ and others. However, it remains an outstanding challenge to control Li stripping/deposition during cycling of LMBs. To address this challenge, understanding the mechanism of Li growth is critical toward achieving the dendrite-free operation of LMBs.

Both the tip-growth and bottom-growth modes of Li dendrites have been reported in the literature. ${ }^{15,16,31-}$ ${ }^{36}$ Dendritic metal deposition can involve the long-range diffusion-limited mechanism associated with a well-known characteristic "Sand's time". ${ }^{31-33}$ In this case, Li dendrites mainly form via the tip-growth mode caused by a severe scarce of $\mathrm{Li}$ ions in liquid electrolytes. When the supply of $\mathrm{Li}$ ions is adequate, the bottom-growth mode can become predominant. During this process, the highly reactive metallic Li usually causes quick formation of a stable SEI film on its surface to prevent further reaction with liquid electrolytes. ${ }^{15,16,34-36}$ Such growth can be strongly influenced by cracking and self-healing of the SEI film on whisker's surface. ${ }^{37}$ On the other hand, protrusion of a Li whisker from the surface of the Li metal anode is usually associated with cracking and self-healing of the SEl film on the surface of bulk Li metal. ${ }^{38,39}$ Despite these mechanistic insights into Li dendrite growth, the dynamic processes of SEI cracking and self-healing have not been clearly understood. Therefore, direct observations of real-time cracking and self-healing of the SEl film are critically needed, in order to understand the mechanism of Li dendrite growth and ultimately find an effective means to control Li dendrite growth. 
In situ experiment is particularly useful for revealing the dynamic structural evolution during the growth of Li whiskers. For example, in situ observations of Li growth in a glass cell optical microscope, $15,16,35$ liquid cell transmission electron microscope (TEM), $36,38,40$ or plastic cell scanning electron microscope $(\mathrm{SEM})^{34,41}$ have been reported to investigate the growth mechanism of Li deposits. However, the nanoscale structural changes such as cracking and self-healing of the ultrathin SEI film are not readily trackable, because of the low resolution of optical microscope and SEM as well as the unsatisfactory image quality of liquid-cell TEM.

Generally, the SEl film consists of a dense inner layer with the main constituents of inorganic $\mathrm{Li}_{2} \mathrm{CO}_{3}, \mathrm{Li}_{2} \mathrm{O}$ and $\mathrm{LiF}$, and a porous outer layer with organic $\mathrm{Li}$ compounds such as $\mathrm{ROCO}_{2} \mathrm{Li}$, ROLi, and others. ${ }^{17,18}$ As the inner layer is directly in contact with metallic $\mathrm{Li}_{1} \mathrm{Li}_{2} \mathrm{CO}_{3}$ and $\mathrm{Li}_{2} \mathrm{O}$ are the important $\mathrm{SEl}$ components that can strongly influence the electro-chemo-mechanical responses of a growing Li deposit. ${ }^{42}$ In situ imaging of the dynamic formation, fracture, reformation of either a $\mathrm{Li}_{2} \mathrm{CO}_{3}$ or $\mathrm{Li}_{2} \mathrm{O}$ film on the surface of a growing Li deposit could provide crucial understanding of dendritic Li growth under the confinement of surface SEI films in real LMBs.

\section{Results}

To observe the dynamic processes occurring in the SEl film of $\mathrm{Li}_{2} \mathrm{CO}_{3}$ on metallic $\mathrm{Li}$, an in situ electrochemical plating device was constructed inside an aberration-corrected environmental transmission electron microscope (ETEM). As shown in Supplementary Fig. 1, a piece of Li metal on a W tip was used as the anode; a $\mathrm{Li}_{2} \mathrm{CO}_{3}$ layer on the surface of $\mathrm{Li}$ metal (formed within a $\mathrm{CO}_{2}$ atmosphere inside ETEM) served as the solid electrolyte; and arc discharged multiwall carbon nanotubes (MWCNTs) acted as the cathode. When an external voltage was applied to this electrochemical plating device, metallic $\mathrm{Li}$ was deposited onto the surface of MWCNTs. Meanwhile, $\mathrm{CO}_{2}$ in the ETEM chamber reacted with freshly deposited $\mathrm{Li}$ to form a surface layer of $\mathrm{Li}_{2} \mathrm{CO}_{3} .{ }^{43}$ The formation of such a $\mathrm{Li}_{2} \mathrm{CO}_{3}$ layer on the deposited $\mathrm{Li}$ is considered to represent similar processes of SEl formation on the surface of Li deposits in liquid organic electrolytes of practical LMBs, which involve electrochemical reactions between Li deposits and electrolytes. As shown in Fig. 1a-b and Supplementary Video 1, when a negative potential was applied to the MWCNT cathode, Li ions from the Li metal anode diffused through the solid electrolyte layer of $\mathrm{Li}_{2} \mathrm{CO}_{3}$ on the surface of the Li metal anode to lithiate the MWCNT. As lithiation of the MWCNT became saturated, a $\mathrm{Li}$ sphere emerged at the contact point of $\mathrm{MWCNT} / \mathrm{Li}_{2} \mathrm{CO}_{3} / \mathrm{CO}_{2}$ via an electrochemical plating process of $\mathrm{Li}^{+}+\mathrm{e}^{-} \rightarrow \mathrm{Li}$. Meanwhile, an SEl film, in the form of a $\mathrm{Li}_{2} \mathrm{CO}_{3}$ shell, formed on the surface of the $\mathrm{Li}$ sphere. This $\mathrm{Li}_{2} \mathrm{CO}_{3}$ shell exhibits darker contrast than the Li core and thus can be recognized during in situ TEM observation. ${ }^{43}$ It was formed via electrochemical/chemical reactions between $\mathrm{Li}$ and $\mathrm{CO}_{2}$ gas. As Li plating proceeded, the $\mathrm{Li}$ sphere grew from a diameter of $200 \mathrm{~nm}$ (Fig. 1b) to $\sim 400 \mathrm{~nm}$ (Fig. 1d), and the thickness of the SEl shell increased accordingly. Under a high magnitude of applied potential $(\sim-1.0 \mathrm{~V})$, the growing Li sphere triggered the formation of a local crack (marked by a yellow arrowhead in Fig. 1e) in the SEI shell. Subsequently, two symmetric cracks 
(marked by a pair of yellow arrowheads in Fig. 1f) appeared in the SEl surface layer near the bottom of the Li sphere, suggesting the formation of a three-dimensional (3D) ring crack in the SEl shell. As a result of SEI cracking, the local mechanical constraint from the SEI shell was released, so that the Li sphere began to elongate along the upright direction (indicated by a yellow arrow in Fig. 1f). Such upright growth (indicated by a yellow arrow in Fig. 1g) continued, resulting in a long and straight Li whisker with a uniform cross-sectional width (Fig. 1 $\mathrm{g}$-j). It is seen that the SEI film formed at the bottom of the Li whisker is markedly thinner than that on the top of this Li whisker (Fig. 1g-j). During the growth of the Li whisker, the top hemispherical head remained unchanged, indicating the major growth occurring near the bottom region of the Li whisker. ${ }^{36}$ Protected by the thin $\mathrm{Li}_{2} \mathrm{CO}_{3}$ shell, the Li whisker was relatively stable under mild electron beam illumination (100 e nm-2 $\left.\mathrm{s}^{-1}\right)$, offering the opportunity to obtain an electron diffraction pattern (EDP) of the electron-beam sensitive Li whisker with little irradiation damage. The EDP of this newly formed Li whisker (Supplementary Fig. 2b) is indexed as the [011] zone axis of the bodycentered cubic (BCC) Li whisker (PDF No. 15-0401) with the $<111>$ growth direction (Supplementary Fig. 2a). The dark field image (Supplementary Fig. 2c) confirms the single crystalline structure of this Li whisker.

The above Li growth process was repeatable with more examples shown in Supplementary Figs. 3, 5-7, Supplementary Videos 2-5. Occasional kinking along single-crystal Li whiskers was observed (Supplementary Figs. 3, S4a). It is interesting to note that even after the kink formation, the whisker remains a single crystal with the same crystallographic direction of growth (Supplementary Fig. 4), which is different from the kinked Li whisker formed in the liquid organic electrolyte ${ }^{44}$. This indicates the preferred growth along a specific crystallographic orientation such as $<111>$ in the BCC Li, considering that there are several equivalent directions within a family of crystallographic orientations. Time lapse monitoring of the whisker growth (Supplementary Figs. 3, 5-6, Supplementary Videos 2-4) shows that in the projected TEM image, the newly formed SEI film was often thinner on one side than on the opposite side before the formation of a kink (Supplementary Figs. 3H, 3J, 5V, 6F). This result suggests that the kink formation can be ascribed to non-uniform Li deposition in the whisker.

The above observations show that the growth of Li whiskers initiated from a 3D ring crack formed in the SEl shell. Such directional growth requires that Li growth along the axial direction of the Li whisker ( $14 \mathrm{~nm} \mathrm{~s}^{-1}$ ) overwhelms self-healing of the ring crack, presumably with the aid of fast reactions between locally exposed $\mathrm{Li}$ and $\mathrm{CO}_{2}$ in the ETEM chamber. We discovered that under a low magnitude of applied voltage $(\sim-0.5 \mathrm{~V})$, the crack in the SEl layer healed quickly, such that no whisker growth was observed, as exemplified in Fig. 2 and Supplementary Video 6. In this case, cracks (marked by red, green, blue and yellow arrowheads) emerged and then healed quickly as the spherical Li particle grew. This growth mode contrasts with that in Fig. 1 where a stable ring crack formed at the bottom of the Li particle, leading to the directional growth of a Li whisker. Note that the healable cracks in Fig. 2 were mainly formed far from the Li source of the Li metal anode in the present electrochemical plating setup. As seen from the dynamic processes of cracking and self-healing in the SEI film (Fig. 2a-d, red arrowheads), cracks formed at the top of the Li particle, due to mechanical damage of the $\mathrm{Li}_{2} \mathrm{CO}_{3}$ shell. Such damage stemmed from 
the buildup of large hoop tensile stresses in the $\mathrm{Li}_{2} \mathrm{CO}_{3}$ shell during expansion of the growing $\mathrm{Li}$ particle. Crack formation in the $\mathrm{Li}_{2} \mathrm{CO}_{3}$ shell exposed the freshly deposited $\mathrm{Li}$ to the $\mathrm{CO}_{2}$ gas in the ETEM chamber. This led to quick local self-healing of the $\mathrm{Li}_{2} \mathrm{CO}_{3}$ shell through the electrochemical reduction of $\mathrm{CO}_{2}$ via $4 \mathrm{Li}+3 \mathrm{CO}_{2} \rightarrow 2 \mathrm{Li}_{2} \mathrm{CO}_{3}+\mathrm{C}$ or $\mathrm{Li}+2 \mathrm{CO}_{2} \rightarrow 2 \mathrm{Li}_{2} \mathrm{CO}_{3}+\mathrm{CO} .{ }^{45}$ Hence, the Li whisker was not formed when the magnitude of applied voltages was low, although two symmetric cracks in the TEM image (indicating a 3D ring crack) were temporarily present, as marked by blue arrowheads in Fig. 2j-I. Apparently, the slow Li deposition provided enough time for the locally-cracked SEI film to self-heal, thus suppressing Li whisker growth. Similar Li deposition processes were observed when applied voltages were low (Supplementary Figs. 8-10 and Supplementary Videos 7-9), demonstrating the repeatability of the growth of spherical Li particles under low driving forces. Moreover, the $\mathrm{Li}_{2} \mathrm{CO}_{3}$-coated Li particles (Fig. 3, Supplementary Fig. 11, and Supplementary Videos 10-11) were obtained when the applied voltage for Li deposition was further reduced to $-0.2 \mathrm{~V}$. These results indicate that the growth of spherical Li particles can be effectively controlled by applied voltage. The thickness of the $\mathrm{Li}_{2} \mathrm{CO}_{3} \mathrm{SEI}$ shell was uniform and gradually increased as the Li particle grew (Fig. 3, Supplementary Fig. 11), indicating the continued reaction of $\mathrm{Li}$ with $\mathrm{CO}_{2}$ to thicken the $\mathrm{Li}_{2} \mathrm{CO}_{3}$ shell.

The above experiments demonstrate that at high applied voltages, Li deposition overwhelms crack selfhealing, leading to the directional growth of Li whiskers; in contrast, at low applied voltages, crack selfhealing is sufficiently fast, leading to the isotropic growth of Li particles. Based on these results, we explored the dynamic control of Li deposit morphologies by tuning applied voltages. An example of the dynamically tunable Li morphology is provided in Fig. 4, Supplementary Video 12. In this case, a Li whisker grew initially under a high applied voltage of $-3.0 \mathrm{~V}$, and this Li deposit subsequently became a nearly sphere (marked by yellow dashed lines in Fig. $4 \mathrm{~b}-\mathrm{h}$ ) after the applied voltage was lowered to $-1.0 \mathrm{~V}$. This result indicates that when self-healing is sufficiently fast, the growth of Li particles predominates. It is also noted that the aspect ratio of another slowly-growing Li whisker (tilted to the left) became smaller because of a low voltage applied (Fig. 4c-h).

We also investigated the impact of SEI composition on the morphology of Li deposits. When the SEI film was composed of $\mathrm{Li}_{2} \mathrm{O}$ rather than $\mathrm{Li}_{2} \mathrm{CO}_{3}$, we observed the favorable growth of $\mathrm{Li}$ particles rather than $\mathrm{Li}$ whiskers. For example, we conducted $\mathrm{Li}$ deposition experiments in an $\mathrm{O}_{2}$ atmosphere, producing the surface SEI film of $\mathrm{Li}_{2} \mathrm{O}$ instead of $\mathrm{Li}_{2} \mathrm{CO}_{3}$. As shown in Fig. 5, Supplementary Fig. 12 and Supplementary Videos 13-14, only spherical Li particles formed even when the applied voltage was high (-1.5 V), which is different from the voltage-dependent formation of $\mathrm{Li}$ whiskers and particles in a $\mathrm{CO}_{2}$ atmosphere. The EDP, dark field image, and electron energy loss spectrum (EELS) (Supplementary Fig. 13) confirmed that the $\mathrm{Li}$ particles formed in $\mathrm{O}_{2}$ gas consist of a $\mathrm{Li}$ core covered with a $\mathrm{Li}_{2} \mathrm{O}$ shell. The $\mathrm{Li}_{2} \mathrm{O}$ layer is usually thicker than the $\mathrm{Li}_{2} \mathrm{CO}_{3}$ layer formed on the surface of Li particles, as shown in Supplementary Fig. 14 . Hence, the dominant growth of spherical Li particles can be attributed to less cracking and faster selfhealing in the SEl film of $\mathrm{Li}_{2} \mathrm{O}$ as opposed to $\mathrm{Li}_{2} \mathrm{CO}_{3}$. An in-depth understanding of these differences 
warrants further study of the electro-chemo-mechanical properties of $\mathrm{Li}_{2} \mathrm{O}$ and $\mathrm{Li}_{2} \mathrm{CO}_{3}$ nanolayers in the future.

\section{Discussion}

Next we consider the effects of Li deposition-induced stress on Li deposit morphologies. By in situ observations of $\mathrm{Li}$ deposition under the mechanical constraint of $\mathrm{Li}_{2} \mathrm{CO}_{3}$ or $\mathrm{Li}_{2} \mathrm{O} \mathrm{SEl}$ films, we find that the growth of Li whiskers and particles shares striking similarities with that of whiskers and hillocks from Sn and $\mathrm{Sn}$ alloy films, ${ }^{46,47}$ since all of these processes are strongly influenced by the need for releasing $\mathrm{Li}$ deposition-induced stresses. Let us consider the SEI shell of $\mathrm{Li}_{2} \mathrm{CO}_{3}$ as an example. In the initial stage of Li deposition, a spherical Li particle was formed. Due to weak mechanical constraints from the ultrathin $\mathrm{Li}_{2} \mathrm{CO}_{3}$ shell, the $\mathrm{Li}$ deposition-induced stresses were low in both the $\mathrm{Li}$ core and $\mathrm{Li}_{2} \mathrm{CO}_{3}$ shell, such that they had a weak impact on the growth of the Li particle. With further Li deposition, continued expansion of the Li particle with concomitant thickening of the $\mathrm{Li}_{2} \mathrm{CO}_{3}$ shell resulted in the buildup of large compressive stresses in the Li particle as well as large hoop tensile stresses in the $\mathrm{Li}_{2} \mathrm{CO}_{3}$ shell. With the occasional occurrence of non-uniform growth, the high stresses in the Li core could result in creep flow 48 of deposited Li (Fig. 6a), given the fact that the room temperature of $295 \mathrm{~K}$ is about $0.65 T_{\mathrm{m}}$, where $T_{\mathrm{m}}$ is the melting point $(455 \mathrm{~K}$ ) of $\mathrm{Li}$. Such creep relaxation of Li deposition-induced stresses smoothed out the local non-uniform growth, resulting in a nearly spherical shape of the Li particle. On the other hand, the buildup of large hoop tensile stresses in the nanocrystalline/amorphous $\mathrm{Li}_{2} \mathrm{CO}_{3}$ shell could cause the formation of defects and even cracks in the shell, so as to release the high stresses induced by continued $\mathrm{Li}$ deposition in both the $\mathrm{Li}$ core and $\mathrm{Li}_{2} \mathrm{CO}_{3}$ shell. ${ }^{46}$

After crack formation in the $\mathrm{Li}_{2} \mathrm{CO}_{3}$ shell, the rate of $\mathrm{Li}$ growth abruptly increased because the resistance to Li deposition from large compressive stresses in the Li core was released substantially, while the driving force for Li deposition from applied voltage was still maintained. The above in situ observations have revealed three distinct Li growth modes (Fig. 6b-d) resulting from the dynamic interplay of cracking and self-healing of the $\mathrm{Li}_{2} \mathrm{CO}_{3}$ surface layer. As shown in Figs. 1k-p and $6 \mathrm{~b}$, when the magnitude of applied voltage is high $(<-1.0 \mathrm{~V})$, the growth of Li whiskers predominates, once a 3D ring crack forms in the $\mathrm{Li}_{2} \mathrm{CO}_{3}$ surface layer. Because of the high voltage and resultant fast rate of $\mathrm{Li}$ deposition, the axial growth is preferably continued in the same cross-sectional region (marked by a red box in the right diagram of Fig. 6b) where the ring crack is initially located. The continued local growth is favored, because the side surface of this cross-sectional region is effectively kept unhealed and thus experiences the least mechanical constraint on axial growth. In addition, as the newly grown cross-sectional layer of $\mathrm{Li}$ moves away from the ring crack region, the $\mathrm{Li}_{2} \mathrm{CO}_{3}$ film gradually grows on the surface of this layer and thus imposes an increasing mechanical constraint on its growth. As a result of continued axial growth in the same cross-sectional region, the growing Li whisker maintains a constant diameter and an unchanged shape of the top part (Fig. 6b, Supplementary Fig. 5y). This mechanism of axial growth is 
further confirmed by our in situ TEM observations, as to be discussed next. Hence, the high voltage and fast Li deposition rate play a critical role in the formation of Li whiskers.

When the applied voltage is in the intermediate range (-0.3 -0.8 V) (Fig. 6c), the growth of Li deposits features several rounds of local extrusion and rounding of Li particles. In this case, the Li particle grows preferentially near the cracked region. Since the rate of Li deposition is lower than the case in Fig. $6 \mathrm{~b}$, the crack in the $\mathrm{Li}_{2} \mathrm{CO}_{3}$ film has sufficient time to heal. Crack self-healing suppresses further local extrusion, resulting in local rounding of Li particles. Growth of a large particle usually involves several rounds of cracking and self-healing, accompanied with repeated processes of local extrusion and rounding. As schematically shown in Fig. $6 \mathrm{c}$, when a local crack is formed in the $\mathrm{Li}_{2} \mathrm{CO}_{3}$ film, $\mathrm{Li}$ growth is localized to the cracked region. Since the rate of $\mathrm{Li}$ deposition is relatively low, creep flow of $\mathrm{Li}$ in this region can smooth the local irregular shape. This smoothing process can be understood in terms of a capillary effect of reducing the local high curvatures of extruded regions. ${ }^{37}$ Moreover, the local crack in the $\mathrm{Li}_{2} \mathrm{CO}_{3}$ film can be healed through reaction between $\mathrm{Li}$ and $\mathrm{CO}_{2}$. Hence, the intermediate voltage and associated intermediate Li deposition rate are responsible for the growth mode with intermittent local extrusion and rounding in the shape of Li particles.

When the applied voltage is in the low range of $-0.1 \sim-0.2 \mathrm{~V}$ (Fig. $6 \mathrm{~d}$ ), the rate of Li deposition is low, so that the Li deposition-induced stress is low. As a result, no major cracks form in the $\mathrm{Li}_{2} \mathrm{CO}_{3}$ layer. Instead, the growing $\mathrm{Li}$ sphere is accommodated by the expanding $\mathrm{Li}_{2} \mathrm{CO}_{3}$ shell. That is, the slow and uniform expansion of the growing $\mathrm{Li}$ sphere results in the low hoop tensile stress and accordingly the uniform expansion in the nanocrystalline/amorphous $\mathrm{Li}_{2} \mathrm{CO}_{3}$ shell, the latter of which likely involves the elastic/plastic stretching as well as the concurrent growth of $\mathrm{Li}_{2} \mathrm{CO}_{3}$ in the shell plane due to continuous reaction between $\mathrm{Li}$ and $\mathrm{CO}_{2}$. As a result, the expanding $\mathrm{Li}_{2} \mathrm{CO}_{3}$ shell can maintain a nearly uniform thickness as the Li sphere grows. Hence, the low voltage and associated low Li deposition rate give rise to the uniform growth of spherical Li particles.

From a further careful tracking of cracked SEls, we found that the continued growth of a Li whisker often occurred at a weak spot along its length where the SEI cracked. Such weak-spot growth contrasts with the generally recognized tip growth and bottom growth. As shown in Fig. 7, the time-resolved TEM observation of a growing Li whisker clearly reveals the weak-spot growth. Figure 7a shows a representative low-magnification TEM image where local SEI cracking (indicated by a red arrow) just occurred at a middle cross section of a growing Li whisker. In the corresponding time series of highmagnification TEM images, it is seen that prior to SEI cracking (Fig. 7b), the Li whisker was covered by a SEl layer (in dark gray) with a uniform thickness of about $10.6 \mathrm{~nm}$. Upon the onset of local SEl cracking (Fig. 7c), both the upper edge (marked by a blue arrow) and the lower edge (marked by a red arrow) of the cracked SEI film were clearly identified. During the subsequent growth of this Li whisker (Fig. 7d-k), the lower edge remained almost fixed, while the upper edge continuously moved upward along the growth direction. Obviously, the growth of the Li whisker occurred predominantly in between the two edges that became increasingly separated. Furthermore, the newly formed thin SEl layer (in dark gray) between the 
two edges exhibited a gradual decrease in thickness from the lower to the upper edge of the cracked SEI film (e.g., see Fig. 7g, k). Such thickness variation indicates the occurrence of continued axial growth of the whisker in its cross-sectional region close to the upper edge of the cracked SEl film. As discussed earlier, the continued local growth at a fixed cross-sectional region is favored, because the side surface of this cross-sectional region is effectively kept unhealed and thus subjected to least mechanical constraints on the axial growth of the whisker.

For the whisker shown in Fig. 7a-k, we measured its axial length versus time, which exhibits a linear relationship, as shown by the blue line in Fig. 7l. Also shown in Fig. 71 is another linear curve measured from a different Li whisker, supporting the generality of the linear time law of growth. This linear relationship indicates that the rate-limiting growth step of the Li whisker should involve some "local" $\mathrm{Li}$ reaction instead of Li diffusion along its axial direction. Based on observations from Fig. 7a-k, the "local" $\mathrm{Li}$ reaction can be reasonably attributed to the process of $\mathrm{Li}$ insertion in a cross section of the Li whisker as illustrated in Fig. 7m-n. In other words, during the axial growth of the Li whisker, Li insertion in a cross section should be effectively treated as a local reaction, while it likely involves the radial Li diffusion within this cross section. For a similar phenomenon of the axial growth of single-crystal Sn whiskers, ${ }^{49}$ it has been suggested that $\mathrm{Li}$ insertion in a cross section occurred through the Bardeen-Herring mechanism of climb of a prismatic dislocation loop. Such a prismatic loop has the Burgers vector perpendicular to the cross section and its climb can result in the axial growth of the whisker. The loop-climbing process involves radial diffusion of atoms in the cross-sectional plane as well as local insertion at the dislocation core. In the same vein, the axial growth of Li whiskers could occur via the Bardeen-Herring mechanism of climb of prismatic loops as well. The detailed atomic-scale process of Li insertion in the cross section of Li whiskers cannot be resolved from the present TEM experiment and warrants further studies via for example cryo-TEM imaging ${ }^{44}$ in the future.

\section{Conclusions}

Our in situ TEM experiments have revealed the dynamic interplay of SEI cracking, self-healing and Li deposition that can critically affect the morphology of Li deposits. Under high applied voltage, the high rate of Li deposition causes SEI cracking that dominates over SEl self-healing, leading to the axial growth of Li whiskers. At low applied voltage, the self-healing rate of cracks becomes sufficiently high compared to the Li deposition rate, leading to the growth of spherical Li particles. We have also demonstrated that it is possible to control the Li deposition morphology by varying SEl constituents and Li deposition rates. With a SEI film of $\mathrm{Li}_{2} \mathrm{CO}_{3}$, the growth of $\mathrm{Li}$ whisker or sphere can be controlled by applied voltage. For a SEl layer $\mathrm{Li}_{2} \mathrm{O}$, the growth of $\mathrm{Li}$ sphere rather than whisker prevails, regardless of the magnitude of applied voltage. We have further found that the continued growth of a Li whisker can occur at a weak spot along its length due to local SEl cracking, which contrasts with the generally recognized tip growth or bottom growth of Li whiskers. Our results highlight the crucial role of applied voltage and SEI in controlling the morphology of Li deposits. Broadly, our work underscores the notion of tailoring Li deposition 
morphology and mitigating Li whisker growth by development of designer SEls and electrolyte additives, as well as by controlling the operating voltage/current.

\section{Methods}

The nanobattery setup for Li electroplating is a two-probe system in a spherical aberration-corrected ETEM, as schematically shown in Supplementary Fig. 1. A MWCNT obtained via an arc-discharge method ${ }^{50}$ was glued to an aluminum rod with silver epoxy and was used as the cathode for $\mathrm{Li}$ electroplating. Metallic $\mathrm{Li}$ on a W tip was used as the $\mathrm{Li}$ anode. The $\mathrm{Li}_{2} \mathrm{CO}_{3}$ or $\mathrm{Li}_{2} \mathrm{O}$ formed on the surface of the metallic $\mathrm{Li}$ under $\mathrm{CO}_{2}$ gas or $\mathrm{O}_{2}$ gas, respectively, in the ETEM chamber served as the solid electrolyte to conduct $\mathrm{Li}$ ions. Both the MWCNT cathode and the Li anode were mounted onto a TEMSTM (Scanning Tunneling Microscopy) holder (Pico Femto FE-F20) inside an argon gas filled glove box. Then the holder was sealed in a custom-made air-tight bag filled with dry argon and transferred to the ETEM. Prior to the experiment, high-purity $\mathrm{CO}_{2}(99.99 \%)$ or $\mathrm{O}_{2}$ was introduced into the ETEM chamber with a pressure of 1.0 mbar.

\section{Declarations}

Data availability. The data are available in the Supplementary Information.

\section{Acknowledgements}

This work was financially supported by the National Key Research and Development Program of China (Nos. 2018YFB0104300), National Natural Science Foundation of China (Nos. 21935009, 51772262, 21406191, 11575154, 51971245, 51802277, 52022088, U20A20336), Fok Ying-Tong Education Foundation of China (No. 171064), Natural Science Foundation of Hebei Province (Nos. B2018203297, B2020203037), Hebei One Hundred Talent Program (No. 4570028), Beijing Natural Science Foundation (2202046), Beijing Natural Science Foundation of China-Haidian Special Project (No. L182065), State Key Laboratory for Mechanical Behavior of Materials, Xi'an Jiaotong University (20192103), State Key Laboratory of Molecular Engineering of Polymers, High-Level Talents Research Program of the Yanshan University (Nos. 00500021502, 005000201) and Hunan Innovation Team (2018RS3091).

\section{Author Contributions}

J.Y.H., Y.F.T. and T.T.Y. conceived and designed the project. L.Q.Z., T.T.Y. and H.L. prepared the sample. T.T.Y., H.L., J.Z.C., H.J.Y., C.C.D. and B.Y.G. carried out the in situ ETEM experiments. J.Y.H., Y.F.T., L.Q.Z., T.Z. and T.D.S. supervised the experiments. L.B.W. and Y.Z. performed computational modelling. J.Y.H., T. Z., Y.F.T., and T.T.Y. wrote the paper. T.T.Y., H.L. and Y.F.T. contribute equally to this work. All the authors discussed the results and commented on the manuscript.

\section{Conflict of interest}

The authors declare no competing interest. 


\section{References}

1 Lin, D., Liu, Y., Cui, Y. Reviving the lithium metal anode for high-energy batteries. Nat. Nanotechnol. 12, 194-206 (2017).

2 Cheng, X.-B., Zhang, R., Zhao, C.-Z., Zhang, Q. Toward safe lithium metal anode in rechargeable batteries: A review. Chem. Rev. 117, 10403-10473 (2017).

3 Tikekar, M. D., Choudhury, S., Tu, Z., Archer, L. A. Design principles for electrolytes and interfaces for stable lithium-metal batteries. Nat. Energy 1, 16114 (2016).

$4 \quad \mathrm{Xu}, \mathrm{W}$. , et al. Lithium metal anodes for rechargeable batteries. Energy Environ. Sci. 7, 513-537 (2014).

5 Albertus, P., Babinec, S., Litzelman, S., Newman, A. Status and challenges in enabling the lithium metal electrode for high-energy and low-cost rechargeable batteries. Nat. Energy 3, 16-21 (2018).

6 Han, F., et al. High electronic conductivity as the origin of lithium dendrite formation within solid electrolytes. Nat. Energy 4, 187-196 (2019).

7 Krauskopf, T., Hartmann, H., Zeier, W. G., Janek, J. Toward a fundamental understanding of the lithium metal anode in solid-state batteries-An electrochemo-mechanical study on the garnet-type solid electrolyte $\mathrm{Li}_{6.25} \mathrm{Al}_{0.25} \mathrm{La}_{3} \mathrm{Zr}_{2} \mathrm{O}_{12}$. ACS Appl. Mater. Interfaces 11, 14463-14477 (2019).

8 Guo, Y., Li, H., Zhai, T. Reviving lithium-metal anodes for next-generation high-energy batteries. Adv. Mater. 29, 1700007 (2017).

9 Zou, Z., et al. Mobile ions in composite solids. Chem. Rev. 120, 4169-4221 (2020).

10 Winter, M., Barnett, B., Xu, K. Before Li ion batteries. Chem. Rev. 118, 11433-11456 (2018).

11 Huo, H., Luo, J., Thangadurai, V., Guo, X., Nan, C.-W., Sun, X. Li2CO3: A critical issue for developing solid garnet batteries. ACS Energy Lett. 5, 252-262 (2020).

12 Wang, X., et al. Stress-driven lithium dendrite growth mechanism and dendrite mitigation by electroplating on soft substrates. Nat. Energy 3, 227-235 (2018).

13 Peled, E. The electrochemical behavior of alkali and alkaline earth metals in nonaqueous battery systems-The solid electrolyte interphase model. J. Electrochem. Soc. 126, 2047-2051 (1979).

14 Aurbach, D. Review of selected electrode-solution interactions which determine the performance of $\mathrm{Li}$ and Li ion batteries. J. Power Sources 89, 206-218 (2000).

15 Steiger, J., Kramer, D., Mönig, R. Mechanisms of dendritic growth investigated by in situ light microscopy during electrodeposition and dissolution of lithium. J. Power Sources 261, 112-119 (2014). 
16 Bai, P., Li, J., Brushett, F. R., Bazant, M. Z. Transition of lithium growth mechanisms in liquid electrolytes. Energy Environ. Sci. 9, 3221-3229 (2016).

17 Wang, A., Kadam, S., Li, H., Shi, S., Qi, Y. Review on modeling of the anode solid electrolyte interphase (SEI) for lithium-ion batteries. NPJ Comput. Mater. 4, 15 (2018).

18 Peled, E., Menkin, S. Review-SEl: past, present and future. J. Electrochem. Soc. 164, A1703-A1719 (2017).

19 Xiao, J. How lithium dendrites form in liquid batteries. Science 366, 426 (2019).

20 Porz, L., et al. Mechanism of lithium metal penetration through inorganic solid electrolytes. Adv. Energy Mater. 7, 1701003 (2017).

21 Kerman, K., Luntz, A., Viswanathan, V., Chiang, Y.-M., Chen, Z. Review-Practical challenges hindering the development of solid state Li ion batteries. J. Electrochem. Soc. 164, A1731-A1744 (2017).

22 Swamy, T., et al. Lithium metal penetration induced by electrodeposition through solid electrolytes: Example in single-crystal $\mathrm{Li}_{6} \mathrm{La}_{3} \mathrm{ZrTaO}_{12}$ garnet. J. Electrochem. Soc. 165, A3648-A3655 (2018).

$23 \mathrm{Li}, \mathrm{W}$., et al. The synergetic effect of lithium polysulfide and lithium nitrate to prevent lithium dendrite growth. Nat. Commun. 6, 7436 (2015).

24 Zheng, J., et al. Electrolyte additive enabled fast charging and stable cycling lithium metal batteries. Nat. Energy 2, 17012 (2017).

25 Adams, B. D., et al. Long term stability of Li-S batteries using high concentration lithium nitrate electrolytes. Nano Energy 40, 607-617 (2017).

26 Shi, P., et al. A highly concentrated phosphate-based electrolyte for high-safety rechargeable lithium batteries. Chem. Commun. 54, 4453-4456 (2018).

$27 \mathrm{Fu}, \mathrm{K}$. , et al. Flexible, solid-state, ion-conducting membrane with 3D garnet nanofiber networks for lithium batteries. Proc. Natl. Acad. Sci. USA 113, 7094 (2016).

28 Zhou, W., Wang, S., Li, Y., Xin, S., Manthiram, A., Goodenough, J. B. Plating a dendrite-free lithium anode with a polymer/ceramic/polymer sandwich electrolyte. J. Am. Chem. Soc. 138, 9385-9388 (2016).

29 Li, N.-W., Yin, Y.-X., Yang, C.-P., Guo, Y.-G. An artificial solid electrolyte interphase layer for stable lithium metal anodes. Adv. Mater. 28, 1853-1858 (2016).

30 Liu, Y., et al. An artificial solid electrolyte interphase with high Li-ion conductivity, mechanical strength, and flexibility for stable lithium metal anodes. Adv. Mater. 29, 1605531 (2017).

31 Brady, R. M., Ball, R. C. Fractal growth of copper electrodeposits. Nature 309, 225-229 (1984). 
32 Grier, D., Ben-Jacob, E., Clarke, R., Sander, L. M. Morphology and microstructure in electrochemical deposition of zinc. Phys. Rev. Lett. 56, 1264-1267 (1986).

33 Bard, A. J., Faulkner, L. R. Fundamentals and applications. electrochemical method. 2, 580-632 (John Wiley \& Sons, Inc. New York, 2001).

34 Tang, C.-Y., Dillon, S. J. In situ scanning electron microscopy characterization of the mechanism for Li dendrite growth. J. Electrochem. Soc. 163, A1660-A1665 (2016).

35 Nishikawa, K., Mori, T., Nishida, T., Fukunaka, Y., Rosso, M., Homma, T. In situ observation of dendrite growth of electrodeposited Li metal. J. Electrochem. Soc. 157, A1212 (2010).

36 Kushima, A., et al. Liquid cell transmission electron microscopy observation of lithium metal growth and dissolution: Root growth, dead lithium and lithium flotsams. Nano Energy 32, 271-279 (2017).

37 Yamaki, J.-i., Tobishima, S.-i., Hayashi, K., Keiichi, S., Nemoto, Y., Arakawa, M. A consideration of the morphology of electrochemically deposited lithium in an organic electrolyte. J. Power Sources 74, 219227 (1998).

38 Mehdi, B. L., et al. Observation and quantification of nanoscale processes in lithium batteries by operando electrochemical (S)TEM. Nano Lett. 15, 2168-2173 (2015).

39 Harry, K. J., Hallinan, D. T., Parkinson, D. Y., MacDowell, A. A., Balsara, N. P. Detection of subsurface structures underneath dendrites formed on cycled lithium metal electrodes. Nat. Mater. 13, 69-73 (2014).

40 Zeng, Z., Liang, W.-I., Liao, H.-G., Xin, H. L., Chu, Y.-H., Zheng, H. Visualization of electrode-electrolyte interfaces in $\mathrm{LiPF}_{6} / \mathrm{EC} / \mathrm{DEC}$ electrolyte for lithium ion batteries via in situ TEM. Nano Lett. 14, 1745-1750 (2014).

41 Orsini, F., et al. In situ SEM study of the interfaces in plastic lithium cells. J. Power Sources 81-82, 918-921 (1999).

42 Shi, S., Qi, Y., Li, H., Hector, L. G. Defect thermodynamics and diffusion mechanisms in $\mathrm{Li}_{2} \mathrm{CO}_{3}$ and implications for the solid electrolyte interphase in Li-ion batteries. J. Phys. Chem. C. 117, 8579-8593 (2013).

43 Yang, T., et al. Air-stable lithium spheres produced by electrochemical plating. Angew. Chem., Int. Ed. 57, 12750-12753 (2018).

$44 \mathrm{Li}, \mathrm{Y}$., et al. Atomic structure of sensitive battery materials and interfaces revealed by cryo-electron microscopy. Science 358, 506 (2017).

$45 \mathrm{Xu}, \mathrm{S} ., \mathrm{Das}, \mathrm{S}$. K., Archer, L. A. The $\mathrm{Li}-\mathrm{CO}_{2}$ battery: a novel method for $\mathrm{CO}_{2}$ capture and utilization. RSC Adv. 3, 6656-6660 (2013). 
46 Sarobol, P., Blendell, J. E., Handwerker, C. A. Whisker and hillock growth via coupled localized Coble creep, grain boundary sliding, and shear induced grain boundary migration. Acta Mater. 61, 1991-2003 (2013).

47 Chason, E., Jadhav, N., Pei, F., Buchovecky, E., Bower, A. Growth of whiskers from Sn surfaces: Driving forces and growth mechanisms. Prog. Surf. Sci. 88, 103-131 (2013).

48 Herbert, E. G., Hackney, S. A., Thole, V., Dudney, N. J., Phani, P. S. Nanoindentation of high-purity vapor deposited lithium films: A mechanistic rationalization of diffusion-mediated flow. J. Mater. Res. 33, 1347-1360 (2018).

49 Lee, B. Z., Lee, D. N. Spontaneous growth mechanism of tin whiskers. Acta Mater. 46, 3701-3714 (1998).

50 Zhang, L., et al. Probing the charging and discharging behavior of $\mathrm{K}^{-} \mathrm{CO}_{2}$ nanobatteries in an aberration corrected environmental transmission electron microscope. Nano Energy 53, 544-549 (2018).

\section{Figures}



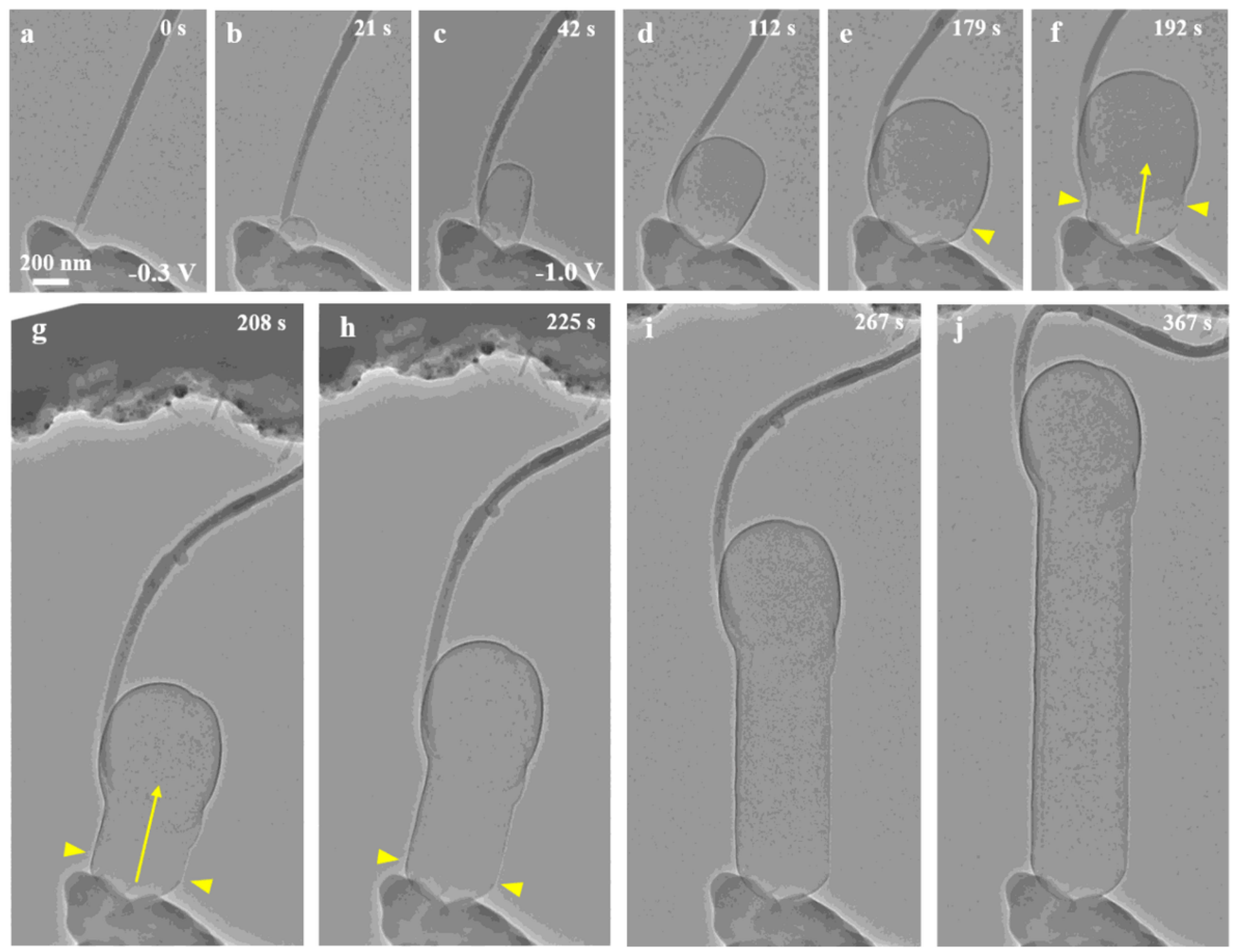

\section{Figure 1}

In situ growth of an electroplated Li whisker at a high applied voltage (-0.3 $\mathrm{V}$ from $a-b,-1.0 \mathrm{~V}$ from $c-j)$ in a CO2 atmosphere. (a-b) Upon applying a voltage at the CNT cathode. A spherical Li particle emerged at the three-phase point. (c-j) With the growth of this Li particle, a local crack (marked by a yellow arrowhead, e) formed in the SEl surface film. When two symmetric cracks (marked by two yellow arrowheads, $\mathrm{f}$ ) appeared in the SEI film, a 3D ring crack was fully formed. As a result, a straight Li whisker grew with the continuous cracking-self-healing cycles at a similar location (indicated by yellow arrowheads, $f-h$ ) near the root of the whisker ( $\mathrm{f}-\mathrm{j})$. 

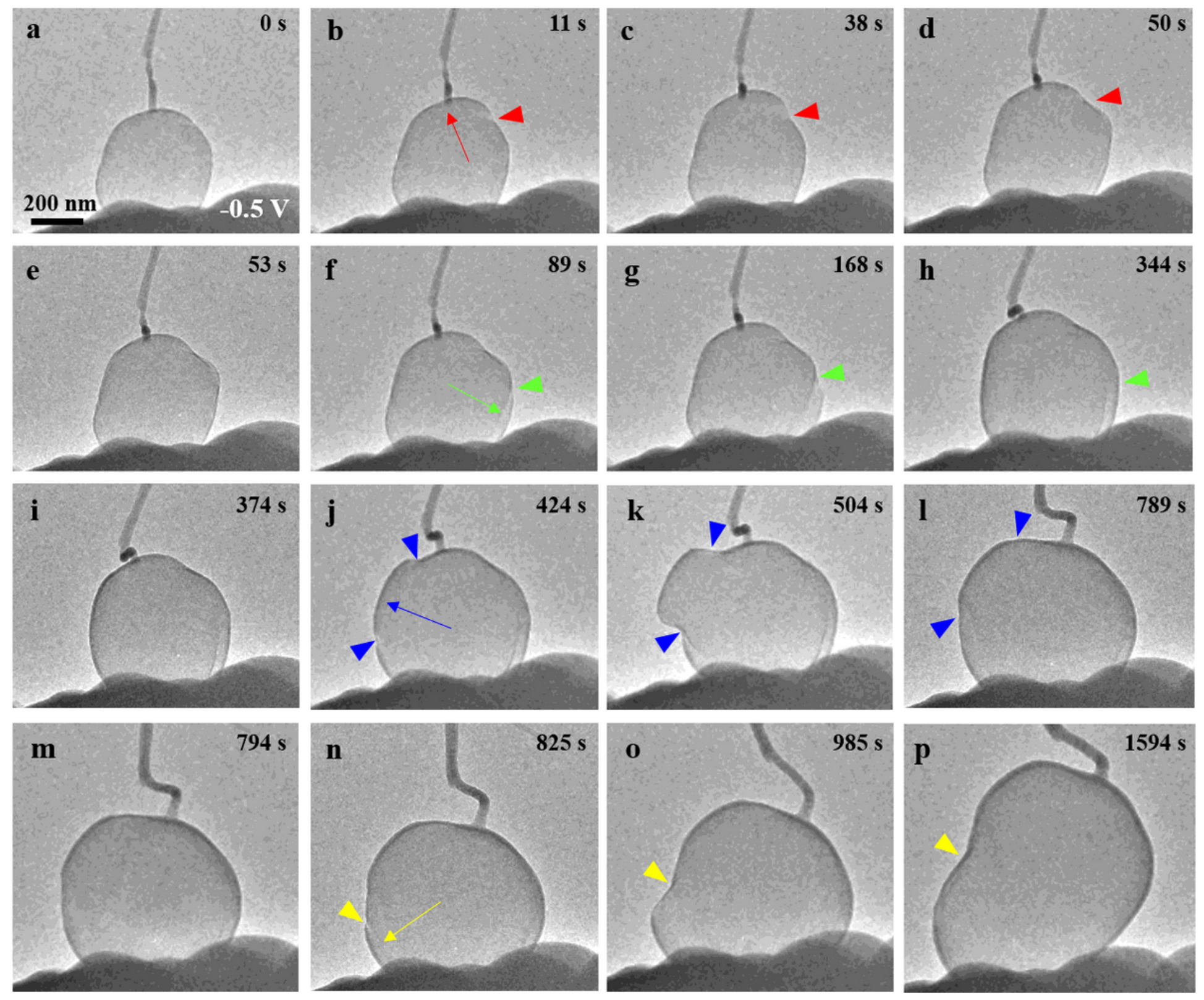

\section{Figure 2}

In situ growth of an electroplated Li particle at a low applied voltage (-0.5 V) in a CO2 atmosphere. (a-d) The first cycle of cracking and self-healing of the SEI shell of Li2CO3. (e-h) The second cycle of cracking and self-healing of the SEI shell of Li2CO3. (i-I) The third cycle of cracking and self-healing of the SEI shell of Li2CO3. $(m-p)$ The fourth cycle of cracking and self-healing of the SEl shell of Li2CO3. The arrowheads indicate the cracks formed, while the arrows indicate the direction of local expansion during the growth of this Li particle. 

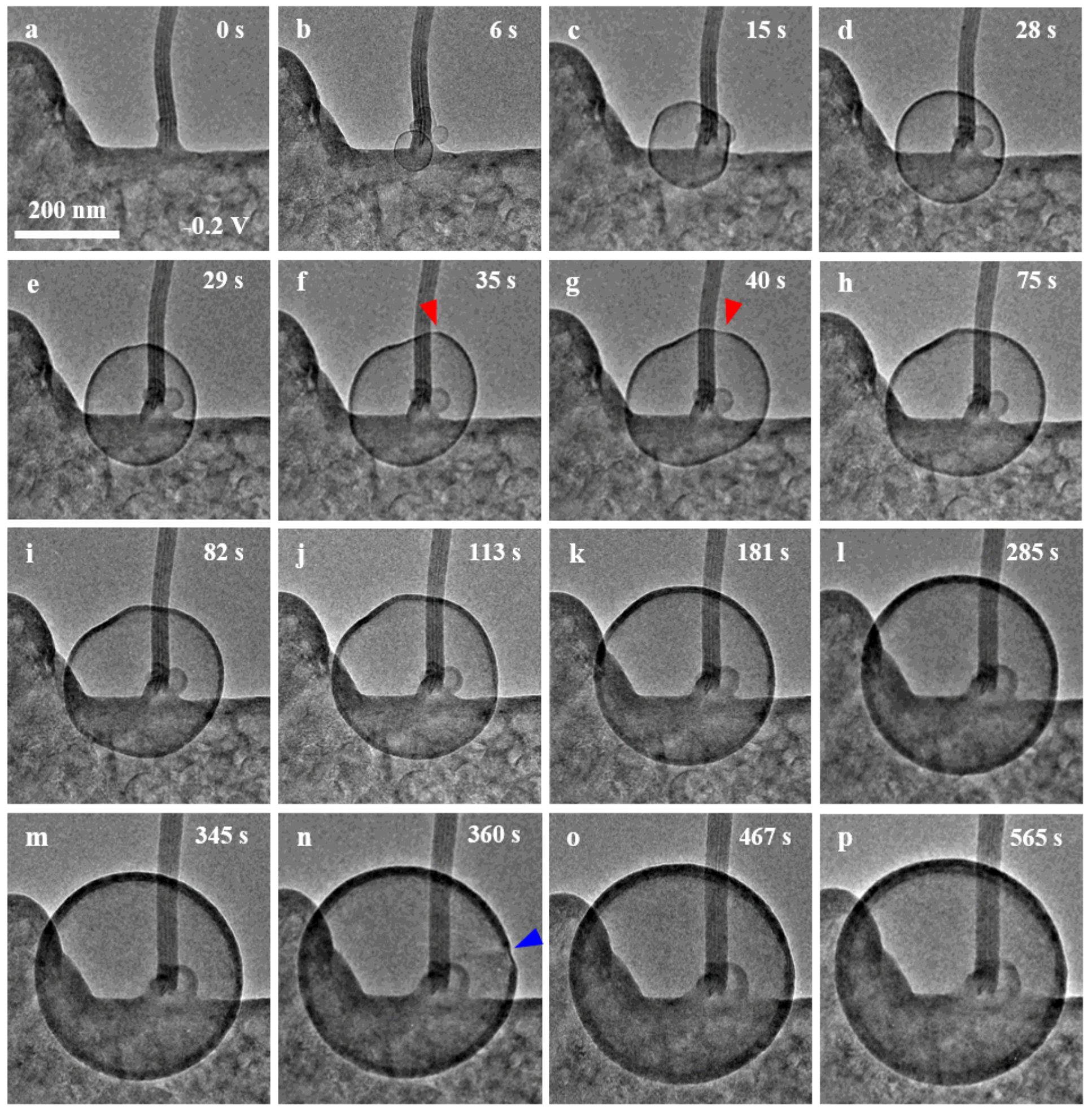

\section{Figure 3}

In situ growth of Li sphere under a low applied voltage (-0.2 V) in a CO2 atmosphere. (a-p) Time lapse TEM images showing the formation of an electroplated Li sphere covered with a SEl shell of Li2CO3. Cracks in the SEI shell were rarely observed during the growth of this sphere. Even if an incipient crack (marked by red and blue arrowheads in $\mathrm{f}-\mathrm{g}, \mathrm{n}$ ) formed, it healed quickly. 

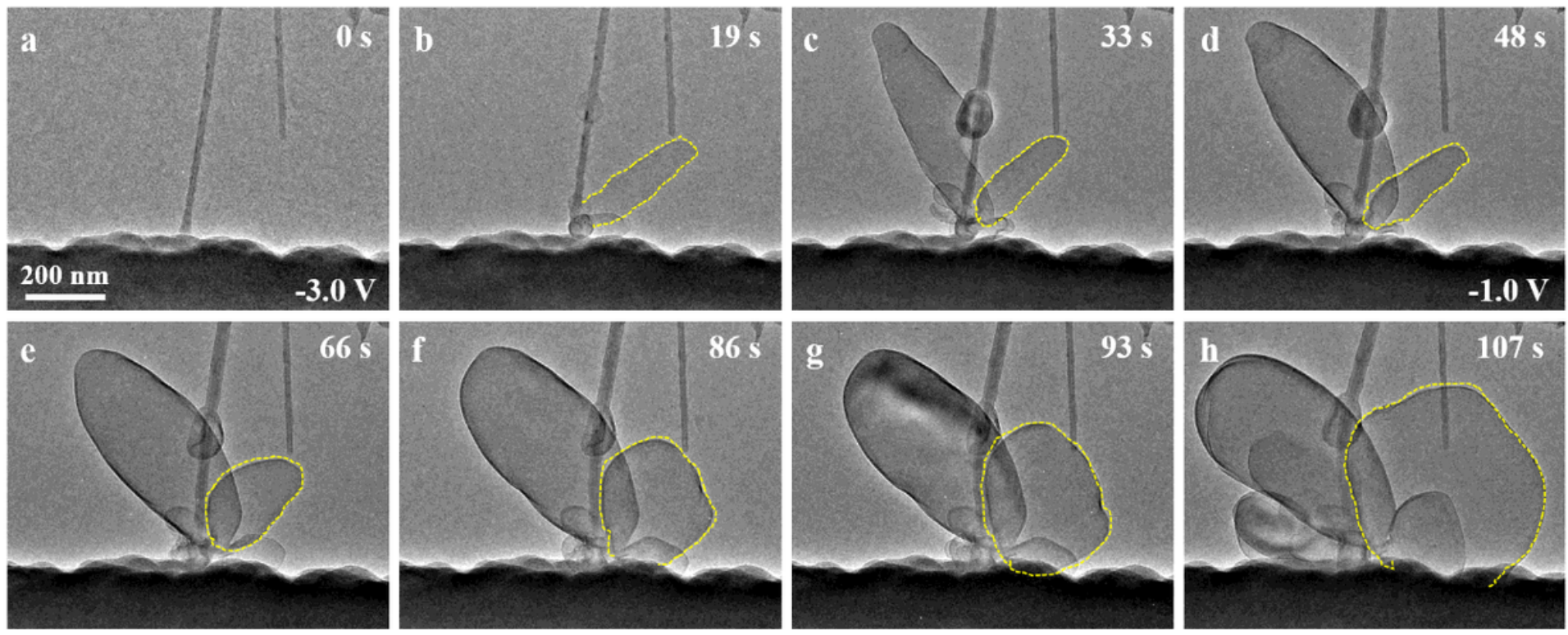

\section{Figure 4}

In situ TEM studies of the transformation of Li whisker into Li sphere in a $\mathrm{CO} 2$ atmosphere (-3.0 V from a$c,-1.0 \mathrm{~V}$ from d-h). (a-h) By decreasing the applied voltage, an initially formed Li whisker was transformed into a nearly spherical Li particle. Yellow dashed lines outline the shapes of Li whisker and particle.
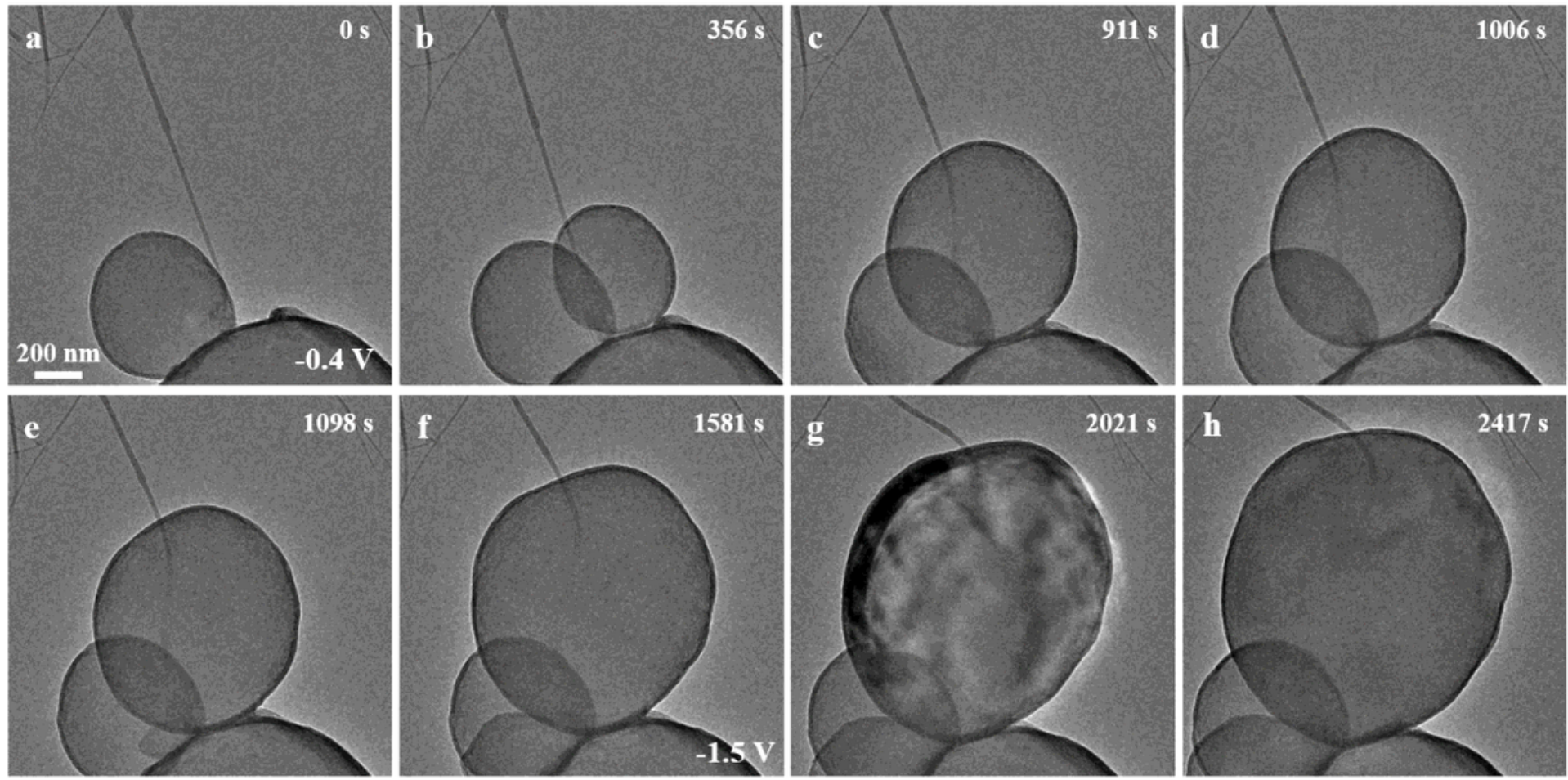

\section{Figure 5}

In situ growth of Li sphere at a high applied voltage in an 02 atmosphere (-0.4 $\mathrm{V}$ from a-e, $-1.5 \mathrm{~V}$ from $\mathrm{f}-\mathrm{h})$. (a-h) Time lapse TEM images showing the formation of two spherical Li particles with a SEI shell of Li2O 
at a high applied voltage in an 02 atmosphere. No cracks were observed during the whole process of $\mathrm{Li}$ deposition.

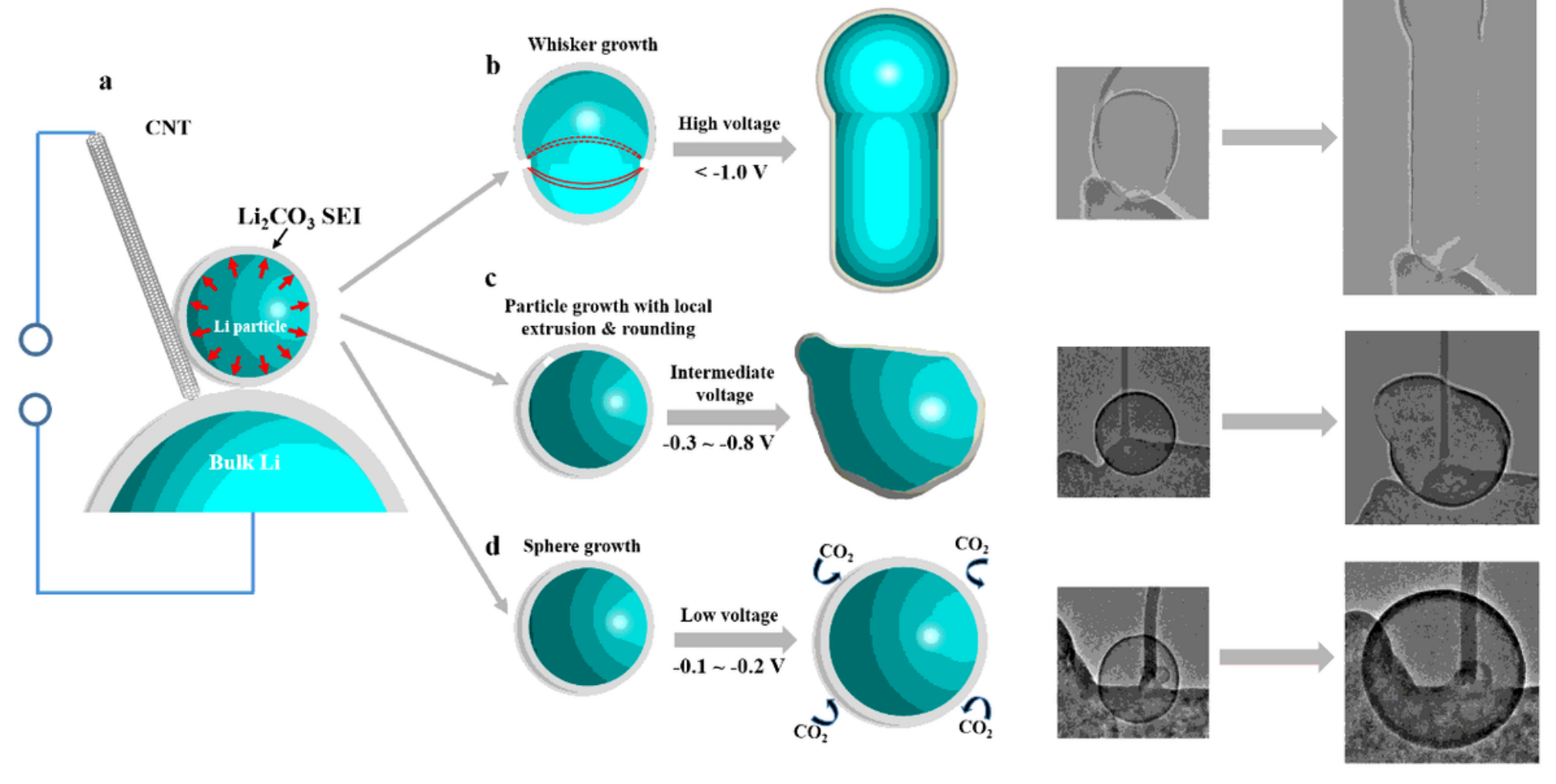

Figure 6

Schematic illustration of three different modes of Li growth governed by the dynamic interplay of cracking/self-healing of SEI and Li deposition rate (controlled by applied voltage). (a) Schematic of the Li plating electrochemical device. (b) Growth of a Li whisker from an initial Li particle under a high applied voltage. A 3D ring crack forms in the SEl shell (with the upper and lower crack edges marked by red lines) of a Li particle (left). Cracking leads to directional growth of a Li whisker. (c) Growth of a Li particle with local extrusion under an intermediate applied voltage. (d) Growth of a spherical Li particle under a low applied voltage. An initially small particle (left) grows into a large particle. 

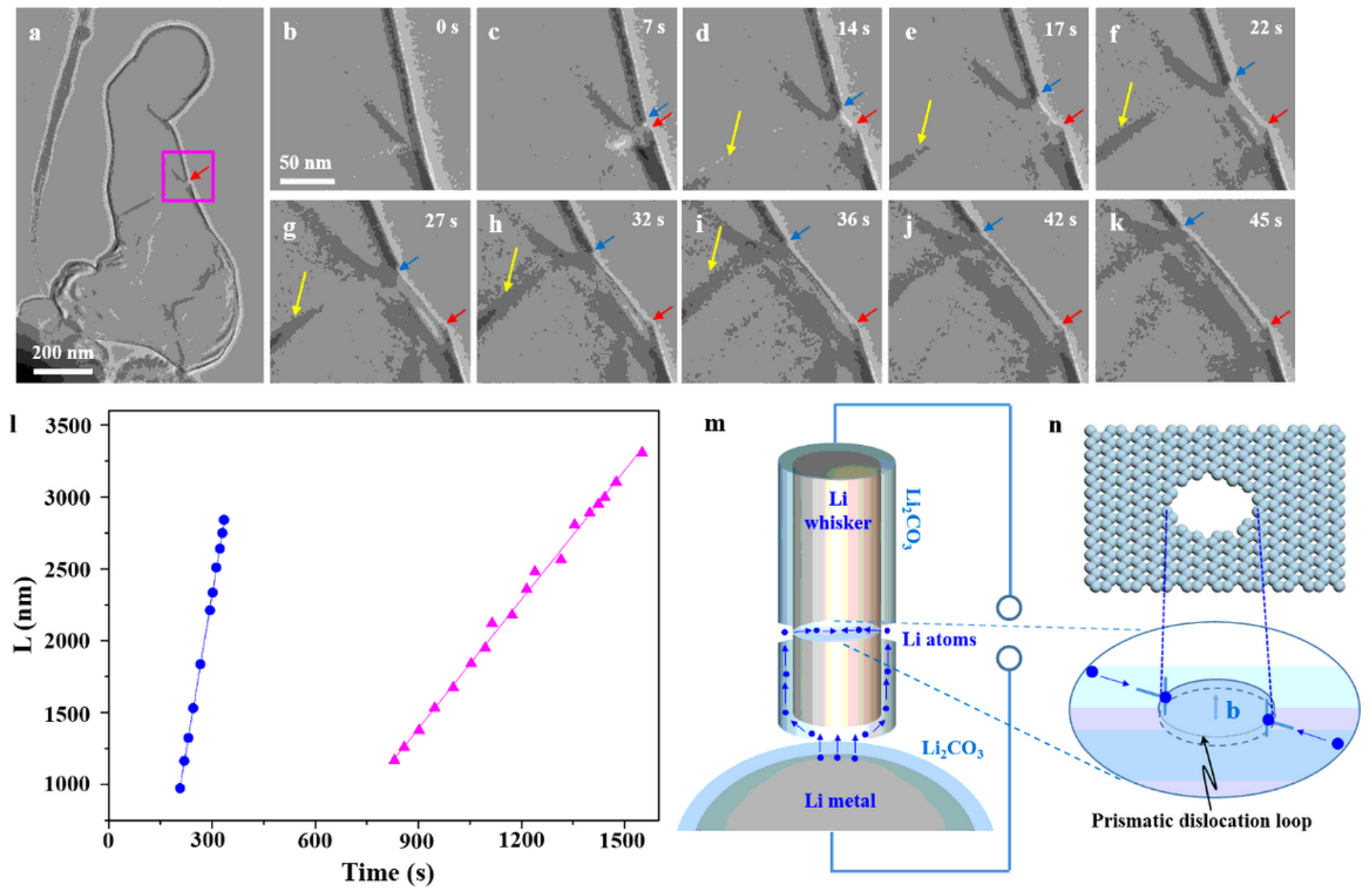

\section{Figure 7}

Time-resolved TEM imaging of the "weak spot" growth mode of a Li whisker, which contrasts with the generally recognized mode of tip growth or bottom growth. (a) A low-magnification TEM image showing the local cracking (marked by a red arrow) in the SEl shell of Li2CO3. (b-k) Time series of highmagnification TEM images showing the axial growth of the Li whisker in between the upper edge (marked by the blue arrow) and lower edge (marked by the red arrow) of the cracked SEl film. (I) Measured length $(\mathrm{L})$ versus time ( $\mathrm{t}$ ) for the Li whisker in (b-k) (blue circles) as well as for another Li whisker (pink triangles). The solid line are linear fitting curves. $(\mathrm{m})$ Schematic illustration showing the sequential processes of $\mathrm{Li}$ diffusion and insertion during the growth of a Li whisker, including Li supply from the Li metal anode, Li ion diffusion through the solid electrolyte Li2CO3 layer covering the Li anode, Li ion diffusion through the SEI Li2CO3 shell covering the Li whisker core, and Li insertion into a cross-sectional region of the Li whisker where the local SEI shell breaks. The last process is considered as the rate-limiting step of "local" reaction during the axial growth of the Li whisker. (n) Schematic illustration of Li insertion in a cross section of the Li whisker through the Bardeen-Herring mechanism of climb of a prismatic dislocation loop. Such a prismatic loop has the Burgers vector $b$ perpendicular to the cross section and its climb via atom insertion at the dislocation core can result in the axial growth of the whisker.

\section{Supplementary Files}


This is a list of supplementary files associated with this preprint. Click to download.

- Videos.zip

- SupplementaryXXinformation.docx 